\title{
INVESTIGACIONES
}

\section{Análisis de las características de la formación de familiares con colectivos vulnerables*}

\author{
Analysis of the characteristics of family education with vulnerable collectives
}

\author{
M. Ángeles Serrano Alfonso ${ }^{a}$, Javier Díez-Palomar ${ }^{b}$, \\ Montserrat Guasch-García ${ }^{c}$ \\ ${ }^{a}$ Universidad Rovira i Virgili, Tarragona \\ Correo electrónico: mariadelosangeles.serrano@urv.cat \\ ${ }^{\text {b } U n i v e r s i d a d ~ d e ~ B a r c e l o n a ~}$ \\ Correo electrónico: jdiezpalomar@ub.edu \\ ${ }^{\mathrm{c}}$ Universidad Rovira i Virgili, Tarragona \\ Correo electrónico: montserrat.guasch@urv.cat
}

\begin{abstract}
RESUMEN
En este artículo se identifican, por un lado, elementos exclusores que dificultan que las familias pertenecientes a colectivos vulnerables (concretamente familias gitanas, inmigrantes y con bajos niveles socioeducativos) participen en los programas formativos ofrecidos por las escuelas de educación primaria. Por otro lado, se identifican cuáles son las características que posibilitan superar estas dificultades, ofreciendo segundas oportunidades educativas a estos colectivos. Entre estos elementos aportados se incide en la importancia de ofrecer una formación de calidad orientada a promover oportunidades educativas y sociales para los y las familiares. Además, se destaca la importancia de fomentar un aprendizaje basado en evidencias científicas, que facilite la acreditación de los conocimientos adquiridos a través de titulaciones, así como la promoción de oportunidades para la conciliación familiar.
\end{abstract}

Palabras clave: educación, familias, organización, diseño, participación

\begin{abstract}
This article identifies, on the one hand, the exclusionary elements that make difficult the participation of families from vulnerable collectives (specifically Roma, immigrant and with low socio-educational levels families) in the educational programmes offered by primary schools. On the other hand, it identifies the characteristics that overcome such difficulties, offering second educational chances to these collectives. Among the provided elements it is stressed the importance of offering a quality learning oriented to the promotion of educational and social opportunities for all relatives. Besides, it is emphasised the importance of promote a learning based on scientific evidences, that facilitates the accreditation of knowledge through valid degrees as well as the promotion of opportunities for family conciliation.
\end{abstract}

Key words: training, families, organization, design, participation

Financiación del proyecto: El proyecto EDU-FAM. Mejora del sistema educativo a través de la formación de familiares de grupos vulnerables (2014-2017) está financiado por la convocatoria 2013 del Programa Estatal de Investigación, Desarrollo e Innovación Orientada a los Retos de la Sociedad, modalidad 1, "Retos Investigación": Proyectos de I+D+I, del Ministerio de Economía y Competitividad. Gobierno de España.. 
De acuerdo con la literatura científica, la formación de familiares se destaca como uno de los aspectos clave que explican el éxito educativo del alumnado. Los estudios previos definen las formas de participación de las familias en el proceso educativo (Flecha, 2015; García \& Ríos, 2014). La investigación de Lastikka y Garcia-Carrión (Lastikka \& GarciaCarrión, 2012) ofrece resultados que sugieren que la forma de participación de familiares que más éxito produce en educación es la que denominan "participación educativa", una de las siete actuaciones educativas de éxito identificadas en INCLUD-ED (Flecha, 2015). Esta "participación educativa" incluye la formación de familiares, donde familias y otros miembros de la comunidad participan en programas educativos que responden a sus necesidades (Flecha, 2015). Sin embargo, a pesar de haber identificado cuál es la forma de participación que mejores resultados produce en términos de éxito académico por lo que a participación de las familias se refiere, aún existe un vacío en cuanto a identificar cuáles son los elementos que potencian (o dificultan) que las familias acaben desarrollando este tipo de actuaciones.

El objetivo de este artículo es ofrecer datos que permitan discutir precisamente esa pregunta de investigación: ¿cuáles son los elementos exclusores que dificultan que las familias pertenecientes a colectivos vulnerables (concretamente familias gitanas, inmigrantes y con bajos niveles socioeducativos) participen en los programas formativos ofrecidos por las escuelas de educación primaria? Y, en consecuencia, ¿cuáles son las características que posibilitan superar estas dificultades, ofreciendo segundas oportunidades educativas a estos colectivos?

\section{ANTECEDENTES TEÓRICOS DE LA FORMACIÓN DE FAMILIARES}

La formación de familiares es una de las actuaciones educativas de éxito que la literatura científica previa ha identificado como parte de lo que se denomina "participación educativa" de las familias y otros miembros de la comunidad educativa (Díez-Palomar, Gatt \& Racionero, 2011; Flecha, 2015; Garcia \& Ríos, 2014; INCLUD-ED Consortium, 2009). De acuerdo con esos estudios, sabemos que no toda forma de participación contribuye a incrementar las posibilidades de que los niños y niñas obtengan buenos resultados académicos (Díez-Palomar \& Kanes, 2012; Peressini, 1998; Sánchez Oñate, Reyes Reyes, \& Villarroel Henríquez, 2016). Además, existe un vacío importante en la literatura por lo que respecta a conocer cuáles son las características que potencian (o dificultan) la formación de familiares de éxito, especialmente por lo que respecta a familiares pertenecientes a colectivos vulnerables tales como población gitana, inmigrante y/o con bajos niveles socioeducativos. Por ese motivo, en este artículo discutimos esta contribución en base a los datos recabados a través del proyecto de investigación EDU-FAM. Mejora del sistema educativo a través de la formación de familiares de grupos vulnerables (20142017). En particular pretendemos sistematizar las evidencias científicas encontradas en esta investigación que nos permitan identificar dichas características.

En 2008, Aubert y sus colegas publicaron un libro titulado Aprendizaje Dialógico (Aubert, Flecha, García, Flecha \& Racionero, 2008). Una de las ideas principales que surgen de este libro, por lo que respecta a la participación y formación de familiares, es que el aprendizaje de los niños y de las niñas pasa no solo por las interacciones que mantengan con el profesorado y el resto de compañeros/as; sino que la relación que puedan mantener 
(tanto dentro como fuera del centro) con otras personas es también una variable clave que explica su aprendizaje. Esta afirmación es coherente con resultados de investigaciones previas en diversos lugares del mundo. Destacan, quizás por el amplio calado que ha tenido después en la comunidad educativa, los resultados publicados por Lev S. Vygotsky durante el primer cuarto del siglo pasado (Vygotsky, 1995; 1996). El investigador ruso acuñó el conocido concepto de zona de desarrollo próximo para referirse a la distancia que existe entre lo que el niño/a sabe y lo que puede llegar a saber, en potencia, con la ayuda de un adulto o de un compañero/a más capaz. A pesar de que la investigación de Vygotsky permaneciera desconocida por espacio de más de medio siglo (hasta que Michael Cole en Estados Unidos tradujese sus principales obras al inglés durante la década de los años sesenta), su paulatina difusión en el ámbito educativo ha encontrado puntos de conexión con el trabajo que otras personas han realizado en el ámbito de la participación de familiares (Aubert et al., 2008).

Actualmente ya nadie discute la importancia de este tipo de participación en los resultados académicos de los niños y de las niñas. Estudios recientes (Díez-Palomar \& Kanes, 2012) superan las afirmaciones sesgadas a las que llegaban los investigadores durante los años 1970s y 1980s, cuando autores como Bowles \& Gintis (1985, v.o. 1976), Baudelot \& Establet (1976) o Ausubel, Novak \& Hanesian (1989) entre otros, afirmaban que las familias de clases sociales deprimidas "no aprecian la escuela", "porque sus hijos/as se van a dedicar a ocupaciones no cualificadas, o incluso ilegales". Este tipo de prejuicios sociales y culturales sustentaron durante esos años afirmaciones tales como que la escuela reproduce las desigualdades sociales de una estructura socio-económica capitalista basada justamente en la desigualdad (Althusser, 1969). Más tarde se pusieron de manifiesto incluso errores metodológicos en la realización de estos estudios, que sus autores tuvieron que reconocer públicamente (Cole, 1988).

Durante los años ochenta, como propuesta "alternativa" a las teorías de la reproducción, Bourdieu propone la idea de capital cultural (Bourdieu \& Passeron, 1977; Bourdieu, 1990). De acuerdo con Bourdieu, las familias son beneficiarias de lo que él denomina capital cultural. Se trata de un fondo de conocimiento acumulado que tiene su origen, justamente, en la clase social (y las oportunidades a las que cada individuo, por pertenecer a una determinada clase social, tiene acceso). La escuela se hace eco de estas oportunidades, legitimando (y premiando) a quienes disponen de ese capital cultural en sus familias. La familia actúa como garante. Los niños y las niñas cuyas familias son académicas, tienen la suerte de tener acceso a una serie de recursos que les facilitan luego, en la escuela, el poder obtener las mejores calificaciones. Esta línea de pensamiento ha sido desarrollada en estudios posteriores (Reardon, 2011; Suizzo \& Stapleton, 2007; De Graaf, De Graaf \& Kraaykamp, 2000), que señalan cómo el capital cultural de la familia tiene un efecto positivo directo sobre los resultados académicos alcanzados por los niños y las niñas en la escuela. Sin embargo, estas afirmaciones parece que entran en contradicción con los casos en los que niños y niñas de clases sociales con capital cultural bajo también obtienen buenos resultados académicos cuando van a escuelas donde existe una importante participación de la comunidad, ya sea de sus propias familias, o de la de familiares de otros niños y niñas de su grupo clase.

Existen ejemplos conocidos internacionalmente que muestran que la participación de las familias tiene un efecto claramente positivo sobre los resultados de los estudiantes, sin importar ni la clase social de origen, ni el supuesto "capital cultural" de la familia. En 
las Comunidades de Aprendizaje eso sucede justamente de esa manera (Aubert, Molina, Schubert \& Vidu, 2017; Díez-Palomar, Gatt \& Racionero, 2011; Gómez, 2002; Gómez, Munté \& Sordé, 2014). Little Village Lawndale High School en Chicago es otro ejemplo de ello (Díez-Palomar \& Kanes, 2012; Gutstein, 2007, 2006; SOJO, 2011). Este tipo de ejemplos, y otros muchos, muestran que la participación de las familias mejora las oportunidades de los niños y de las niñas, tal y como confirman los resultados de diversos estudios en el ámbito (Boethel, 2004; Desforges, 2003; Driessen \& Smit, 2007; Epstein et al., 2002). Esto ocurre cuando la participación y las oportunidades para participar son verdaderamente democráticas, porque, de hecho, no toda forma de participación produce los mismos efectos (Peressini, 1998).

En 2005, Hoover-Dempsey y Sandler escribían que las familias se involucran en la educación de sus hijos e hijas porque les quieren ayudar a obtener los mejores resultados académicos (Hoover-Dempsey \& Sandler, 2005). Al hacerlo adoptan diferentes formas de participación. Estos autores distinguen entre encouragement, modeling, reinforcement e instruction, como formas básicas de cómo las familias se involucran en la formación de sus hijos/as. Hay tanto familias que pagan maestros/as privados para reforzar los aprendizajes de sus hijos/as, como familias que se centran en animar a sus hijos/as a sacar buenas notas, como familias que deciden ellas mismas "volver a la escuela" para aprender aquellos conocimientos que les van a ayudar luego, en casa, a apoyar a sus hijos/as en su propio proceso de aprendizaje. En Europa, entre 2006 y 2011, se llevó a cabo la investigación más importante y con más recursos financiada por la Comisión Europea: INCLUD-ED, Strategies for inclusión and social cohesion form education in Europe, dirigida por el prof. Flecha (Flecha, 2015). Esta investigación sirvió para identificar lo que se conoce como actuaciones educativas de éxito. Una de las actuaciones educativas de éxito identificadas fue la participación de las familias, y, en concreto, la formación de éstas.

En INCLUD-ED se distinguen cinco formas diferentes de participación de las familias: informativa, consultiva, decisiva, evaluativa y educativa (Flecha, 2015; García \& Ríos, 2014). La formación de familiares corresponde al último tipo, la participación "educativa" de las familias, que, de acuerdo con las evidencias encontradas a lo largo de la investigación, es la que más impacto tiene sobre los resultados académicos y la convivencia en las escuelas de los estudiantes. Resultados obtenidos en estudios internacionales ${ }^{1}$ corroboran esta conclusión, también confirmada en los talleres de matemáticas para familias llevados a cabo en diversas regiones como Estados Unidos (Civil \& Andrade, 2003; Civil, 2007; González, Andrade, Civil \& Moll, 2001), Cataluña (Díez-Palomar y Kane, 2012), en reuniones de familiares en la escuela (Castelli \& Pepe, 2008), en seminarios o charlas dirigidas a las familias y a su formación o sensibilización (Warren, Hong, Rubin \& Uy, 2009), o en la involucración de otro tipo de actividades formativas, como por ejemplo las Parent Nights en Finlandia (Gatt, Ojala \& Soler, 2011).

Las líneas actuales de investigación sobre formación de familiares parecen sugerir que esta actuación educativa de éxito contribuye a superar las limitaciones del concepto de capital cultural acuñado por Bourdieu, puesto que la formación previa de las familias,

\footnotetext{
Algunos de los más recientes incluyen: CEMELA - Center for the Mathematics Education of Latinos/as, financiado por la NSF en Estados Unidos, con el "Community and Parents" study group, liderado por la prof. Civil y coordinado por Díez-Palomar y Menéndez; FAMA - Family Math for Adult Learners, financiado por la Unión Europea, dirigido por el Dr. Díez-Palomar; Formació de familiars per a una escola inclusiva, y Formació de professorat per a una educació matemàtica de familiars en contextes multiculturals, financiados ambos por el Gobierno catalán; entre otros.
} 
a pesar de ser una variable relevante como predictor del éxito educativo de los niños/as, no es una variable del tipo sine qua non; la formación de familiares es una alternativa que usan muchas familias para adquirir ese capital cultural al que no tuvieron acceso antes, y que pasa a formar parte de su núcleo familiar, incluidos los hijos/as. Esta contribución se une a otras descubiertas en INCLUD-ED, como el hecho de que la formación previa de la persona no es barrera para su participación activa en actuaciones educativas, como los grupos interactivos, por ejemplo (Valls \& Kyriadides, 2013). Que una familia no participe en la escuela, no condena a los hijos/as de esa familia a no obtener buenos resultados; la participación de la comunidad ofrece a todos los niños y niñas, no solo a los de las familias que participan, la oportunidad de contar con apoyos y ayudas que de otra manera seguro que no tendrían. La formación de familiares es una concreción de esa participación educativa de la comunidad identificada por INCLUD-ED (Flecha, 2015). Lo que la investigación no ha hecho todavía, y ese es el objetivo de este artículo, es identificar cuáles son las barreras y cómo se superan (los elementos transformadores) para que esa formación sea una actuación educativa de éxito.

\section{METODOLOGÍA Y PROYECTO}

EDU-FAM es una investigación educativa financiada por el Plan Nacional I+D+I del Gobierno de España y desarrollada a lo largo de tres años (2014-2017) que ha profundizado en las características de la formación de familiares, analizando tanto los elementos exclusores como los transformadores que fomentan la implementación de esta actuación educativa de éxito entre personas adultas pertenecientes a grupos vulnerables de diferentes zonas geográficas de España. Entre los colectivos vulnerables, EDU-FAM se ha centrado concretamente en familias gitanas, inmigrantes y con bajos niveles socioeducativos, por ser los grupos que presentan una mayor exclusión social en el contexto español. EDU-FAM es, por lo tanto, una investigación clave para contextualizar y presentar aquellos elementos que permitan alcanzar el objetivo de este artículo.

EDU-FAM PROJECT ha llevado a cabo análisis teórico y empírico partiendo de una orientación metodológica comunicativa (Gómez \& Munté, 2015; Soler-Gallart, 2017). La metodología comunicativa pretende analizar y validar un tipo de explicación de los fenómenos, situaciones o interacciones que se producen en la sociedad, mostrando especial atención a aquello que provoca la exclusión social y los elementos que la transforma. Pero, además, la metodología comunicativa facilita que el análisis de la realidad siempre se realice vinculado a la utilidad social de la investigación, esto es, con el objetivo de conseguir una transformación social (Gómez, Puigvert \& Flecha, 2011).

La metodología comunicativa se caracteriza por incorporar el conocimiento, las interpretaciones, reflexiones de todos los participantes en la investigación, ya sean investigadores/as como los propios sujetos de la investigación (Soler-Gallart, 2017). Para ello, la metodología comunicativa establece estrictas condiciones y criterios de procedimiento, tanto durante la recogida de la información (a través del diseño de los instrumentos de trabajo) como en su posterior análisis (Gómez, Puigvert \& Flecha, 2011). Tal y como Soler-Gallart (2017) destaca, el diálogo intersubjetivo entre personas "expertas" y destinatarias es uno de los elementos fundamentales para favorecer la construcción conjunta del conocimiento en la metodología comunicativa. 
Tanto el diálogo intersubjetivo como la consecuente construcción conjunta de conocimiento son imprescindibles en la investigación con grupos vulnerables para obtener un impacto potencial que mejore y transforme su realidad social. Sin embargo, actualmente todavía se llevan a cabo muchas investigaciones sobre grupos vulnerables que no cuentan con las voces de las personas pertenecientes a ellos, debilitando su impacto potencial. Por ejemplo, en el caso de la comunidad gitana, ésta rechaza aquellas investigaciones que solo tratan de obtener información para después extraer sus propias conclusiones, sin contar para nada con ellas y ellos (García-Espinel, 2015; Gómez \& Munté, 2015). La investigación basada en la metodología comunicativa rompe con esta jerarquía metodológica tradicional implícita en los enfoques etnocentristas y relativistas, permitiendo un análisis orientado a la superación de la desigualdad y la exclusión social que facilita el pasar de una investigación "sobre" los colectivos vulnerables a realizar una investigación "con y para" los grupos vulnerables (Soler-Gallart, 2017).

Para poder vehicular esta participación a lo largo de la investigación, el proyecto EDUFAM ha contado con un Consejo Asesor, esto es, un mecanismo de organización de la investigación siguiendo la orientación comunicativa (Gómez, Puigvert \& Flecha, 2011). Este Consejo Asesor ha estado compuesto por familiares perteneciente a grupos vulnerables, alumnado de las escuelas que han participado en el proyecto, personas voluntarias en las acciones de formación de familiares y personal del equipo investigador. Entre las funciones de este órgano asesor se pueden destacar su aporte de conocimientos, el seguimiento y orientación en el desarrollo del proyecto, la revisión crítica de los instrumentos de recogida de datos, el control de que éstos sean comunicativos, la validación y evaluación de toda la investigación y, especialmente, que los resultados obtenidos contribuyan a mejorar la situación de los colectivos a los que va dirigido el proyecto.

La metodología comunicativa ha sido aplicada con éxito en investigaciones europeas tales como el ya mencionado proyecto integrado del sexto Programa Marco INCLUDED: Strategies for inclusion and social cohesion in Europe from education (2006-2011) o el proyecto RTD perteneciente al quinto Programa Marco WORKALÓ: The creation of new occupational patterns for cultural minorities: the gypsy case (2001-2004). En ambos proyectos, el uso de este enfoque metodológico ha producido un impacto relevante no solo a nivel científico, sino también a nivel social y político. Entendemos por impacto social las mejoras sociales logradas como consecuencia de la ejecución de un proyecto o acción particular (Flecha, Soler \& Sordé, 2015; Reale et al., 2017) e impacto político como las repercusiones institucionales de la implementación de ese proyecto o acción (Reale et al., 2017; SIOR, 2016). Así, en el caso de los proyectos referenciados, sus resultados se han recogido en diferentes resoluciones legislativas que incluyen las emitidas por el Parlamento Europeo, las Cortes Generales españolas o el Parlamento de Cataluña (GarcíaEspinel, 2015; Soler-Gallart, 2017).

Bajo esta orientación metodológica EDU-FAM ha efectuado, en primer lugar, una revisión de las bases teóricas e investigaciones internacionales sobre la formación de familiares en grupos vulnerables y las características para su implementación exitosa. En segundo lugar, ha llevado a cabo 8 estudios de caso seleccionados entre centros educativos ubicados en diferentes áreas geográficas del estado español y donde se están llevando a cabo formación de familiares con evidencias de impacto. Todos estos centros atienden a población y alumnado de grupos vulnerables, con especial mención a familias gitanas. En los estudios de caso se han utilizado diferentes técnicas cualitativas y cuantitativas 
de orientación comunicativa (Gómez, Puigvert \& Flecha, 2011) tales como cuestionarios $(\mathrm{N}=161)$, entrevistas semiestructuradas $(\mathrm{N}=95)$, relatos de vida $(\mathrm{N}=13)$ y grupos de discusión $(\mathrm{N}=36)$ con familias, alumnado y profesionales para identificar, mediante la triangulación de los datos, los elementos que facilitan que las familias participen en programas de formación, así como los que la dificultan. Los estudios de caso contemplaban, además, la realización de un análisis longitudinal a un/a familiar participante en cada uno de los centros educativos seleccionados para profundizar en el estudio del impacto de la formación de familiares a lo largo del tiempo. En tercer y último lugar, los resultados obtenidos se han complementado con dos estudios de caso de experiencias internacionales de éxito.

El análisis de la información se ha realizado siguiendo las premisas propias de la metodología comunicativa (Gómez, Puigvert \& Flecha, 2011). Así, se ha diseñado una matriz de análisis en las que se han incluido las dimensiones exclusora y transformadora para 8 categorías (tabla 1). La dimensión exclusora ha englobado las barreras o características de los programas educativos que dificultan a los grupos vulnerables obtener una formación de calidad. También se han incluido aquí las barreras o características de las ofertas educativas que no generan un impacto positivo en la mejora de su situación educativa y social de las personas participantes y sus familias. En contraposición, y como dimensión transformadora, se han contemplado aquellos elementos o características que están potenciando la participación de familiares vulnerables en ofertas formativas de calidad y que incrementan sus oportunidades educativas y sociales, como consecuencia. Las 8 categorías de análisis establecidas se relacionan con los diferentes objetivos planteados en el proyecto y son las siguientes: 1) Características de la formación; 2) Características contextuales; 3) Participación y motivación; 4) Impacto en el aprendizaje; 5) Impacto en la esfera personal; 6) Impacto en la esfera familiar; 7) Convivencia; y 8) Otros.

Tabla 1. Matriz de análisis de los datos

\begin{tabular}{|l|c|c|}
\hline Categorías de análisis & Dimensión Exclusora & Dimensión Transformadora \\
\hline Características de la formación & {$[1]$} & {$[2]$} \\
\hline Características contextuales & {$[3]$} & {$[4]$} \\
\hline Participación y motivación & {$[5]$} & {$[6]$} \\
\hline Impacto en el aprendizaje & {$[7]$} & {$[8]$} \\
\hline Impacto en la esfera personal & {$[9]$} & {$[10]$} \\
\hline Impacto en la esfera familiar & {$[11]$} & {$[12]$} \\
\hline Convivencia & {$[13]$} & {$[14]$} \\
\hline Otros & {$[15]$} & {$[16]$} \\
\hline
\end{tabular}


En el presente artículo recogemos los principales hallazgos del análisis de los estudios de caso relacionados con la primera categoría, características de la formación, aportando evidencias de los elementos exclusores y transformadores que muestran qué facilita y qué dificulta que las familias participen en programas de formación.

\section{ELEMENTOS CLAVE EN LA FORMACIÓN DE FAMILIARES DE ÉXITO}

El análisis de los datos ha identificado cuatro elementos claves entre las características que deben tenerse en cuenta a la hora de implementar programas de formación de familiares dirigidos a colectivos vulnerables. En concreto, hablamos de (1) fomento del aprendizaje instrumental, (2) acreditación de los conocimientos adquiridos a través de titulaciones, (3) formación basada en evidencias científicas y (4) promoción de oportunidades para la conciliación familiar.

\subsection{FOMENTO DEL APRENDIZAJE INSTRUMENTAL}

Lo que Flecha (2000) denomina como "aprendizaje instrumental" dentro de los siete principios que caracterizan el Aprendizaje Dialógico constituye un componente fundamental que explica la participación o no participación en actividades de formación por parte de las familias.

Los datos obtenidos en el trabajo de campo sugieren que existen algunos elementos que podemos considerar como exclusores puesto que dificultan que las familias vulnerables se vinculen a los programas formativos como, por ejemplo, interacciones entorno al aprendizaje que ridiculizan a las personas adultas que deciden retomar su formación o factores personales como un auto-concepto académico negativo. En la siguiente cita, correspondiente a un maestro, se identifican algunos de los prejuicios que presentan los y las familiares en relación a su participación en los cursos de formación de familiares:

El escollo grande es que no se sientan como ridículos por venir a formación, que la formación no los identifica como personas ignorantes. Entonces yo vengo aquí al colegio porque no sé... pues no, yo vengo aquí al colegio porque quiero saber más (Alberto ${ }^{2}$, maestro).

Una de las características que ha posibilitado atenuar este tipo de interacciones es ofrecer a los y las participantes altos niveles de aprendizaje instrumental en las diferentes actividades de formación. En la siguiente cita una de las maestras entrevistadas hace referencia a cómo a través de la participación de algunos familiares en cursos de educación matemática se han contrarrestado las dinámicas contextuales descritas anteriormente. Concretamente, se han atenuado algunos de los miedos que presentaban las personas participantes a la hora de acceder a la formación y se ha incrementado su motivación hacia el aprendizaje.

Este año ha motivado mucho el tema de la formación del [método] ABN porque vi que el año pasado vino gente y porque vi además que era una actividad muy transformadora

\footnotetext{
Todos los nombres que aparecen en las citas de este artículo son ficticios y protegen el anonimato de las personas que los han realizado.
} 
del entorno. Porque es ponernos a pensar, desmitificar y quitar el miedo, pero es que matemáticas la formación no solo del barrio sino de los maestros y voluntarios (Victoria, familiar).

Estos resultados coinciden con los datos obtenidos a partir de los cuestionarios, en donde los participantes valoran la utilidad de la formación recibida para la vida cotidiana. Como se observa en la Figura 1, los participantes indican que la formación les ha servido para aprender habilidades básicas (20\%) y para aprender en general (19\%). Ambas categorías suman un $39 \%$, lo cual indica su relevancia para los participantes.

Figura 1. Utilidad de la formación para la vida cotidiana

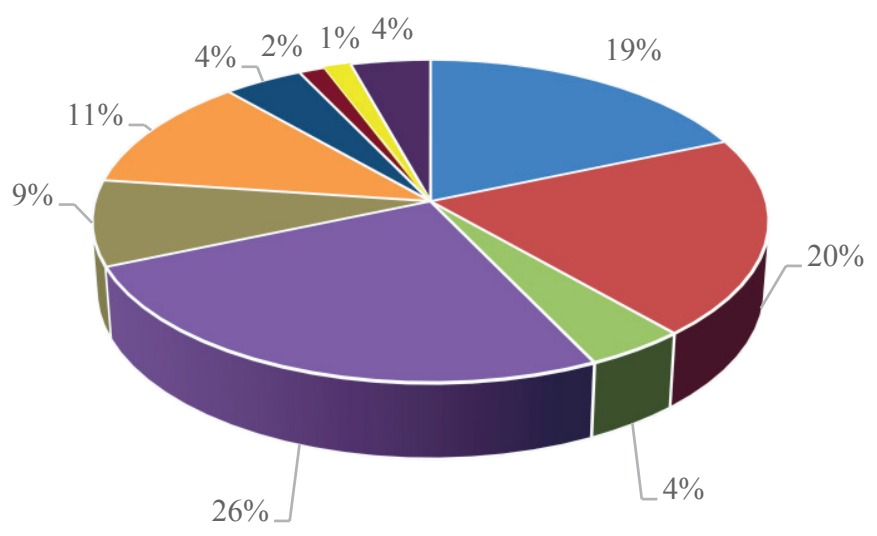

Aprender en general

Aprender habilidades básicas (leer, escribir, multiplicar)

Aprender habilidades sociales (trabajo en equipo, comprensión del otro)

Ayudar (a los hijos en los deberes de la escuela)

Comunicación con otros

Conseguir trabajo

Mejora personal

Motivación

Satisfacción personal

Seguir estudiando 
La preocupación por aportar un aprendizaje de calidad es percibida por los propios participantes en la formación como un elemento positivo. Por ejemplo, en la siguiente cita una de las madres participantes relaciona el mantenimiento de un nivel de aprendizaje adecuando con sentirse bien atendidas y con una implicación por parte del profesorado.

Se preocupan mucho por nosotros los profesores de aquí. Ellos quieren que aprendamos mucho ¿sabes? Y esto es un nivel muy bueno para nosotros. (Valentina, familiar)

Parte del profesorado y miembros de los equipos directivos entrevistados han destacado la importancia de la evaluación y de recoger la evolución realizada por las personas participantes en la formación. En la siguiente cita, una de las profesoras entrevistadas menciona la importancia de la evaluación tanto para guiar su propia acción pedagógica como para corresponsabilizar y concienciar a los educandos de sus progresos educativos.

Me hubiera gustado enterarme más de los resultados, me gustaría ver o saber, por ejemplo, en castellano oral, el nivel del que parten y el nivel que tienen cuando acaban. De esta manera los maestros nos sentiríamos más copartícipes y de esta manera sabríamos el impacto. También es importante que esto conste por escrito. Es importante que la mujer que venga a aprender se lo enseñes [...]. Ellas tienen que ser conscientes de su propia evolución, igual que a los niños les das las notas, darles algo para que ellas también vayan viendo su propia evolución, esto las motivará aún más (Elena, maestra).

\subsection{ACREDITACIÓN DE LOS CONOCIMIENTOS ADQUIRIDOS A TRAVÉS DE TITULACIONES}

Diversos familiares entrevistados han destacado la importancia que desde los centros educativos se impartan cursos oficiales que les den acceso a titulaciones oficiales. De hecho, éste es uno de los elementos clave para comprender su motivación a inscribirse y finalizar los cursos formativos impartidos en la formación de familiares, tal y como se constata en la siguiente cita de una de personas participantes:

Es importante que nos den el papel, porque así podemos enseñar que hacemos algo y no estamos sentadas en casa (Carmen, familiar).

Además, durante las entrevistas se estableció una relación clara entre la obtención de estas titulaciones y las mayores posibilidades de acceder al mercado laboral. Como se indica en la siguiente cita de una de las familiares, la obtención de una titulación es especialmente relevante en el caso de muchas de las personas pertenecientes a colectivos vulnerables que han participado en la investigación educativa de EDU-FAM, ya que, a pesar de tener una larga experiencia en diversos ámbitos laborales, muchas de ellas no disponen de ninguna titulación que acredite dichos conocimientos:

Tú quieres ser camarera de piso, pues tienes que tener un curso de la Junta de Andalucía sobre camarera de piso, con sus horas... si no haces esos cursos esto no te sirve de nada ...Te frustra, te dices que das el perfil, que eres trabajadora, pero yo no lo tengo, te frustras (Isabel, familiar). 
Adelaida, maestra en un centro de formación de adultos, confirma esta misma idea. La vinculación entre certificación de los estudios, del nivel educativo o de las competencias adquiridas a lo largo de la vida es un elemento clave, porque sin dicha certificación, aunque la persona muestre que tiene las competencias que se piden para desempeñar la actividad en el lugar de trabajo, no es suficiente para obtener un contrato laboral. Esto es especialmente dramático en situaciones de crisis, como la reciente crisis económica por la que hemos atravesado, y que ha afectado especialmente a las personas de los colectivos más vulnerables:

La crisis, el ir a buscar un trabajo y al no tener un título no poder optar a ese trabajo. Este año han venido unas chicas jóvenes a apuntarse y lo primero que han dicho es que si no tienen la ESO no las cogen a ningún sitio y son exalumnas de aquí, entonces ven la necesidad que para trabajar tienen que tener un mínimo y en este caso es el certificado (Adelaida, maestra)

El tener una certificación no solo avala una serie de competencias. También proporciona mayor sentimiento de seguridad (empoderamiento) a las personas que consiguen obtener dicha certificación. Isabel lo muestra en el siguiente fragmento de la entrevista que le hicimos:

Sí, porque tú tienes más seguridad y ya con el certificado yo ya sé de sumas, de restas, de multiplicaciones, de dividir, entonces, aunque no tenga la carrera, yo me atrevo, yo me atrevo porque sé que no voy a ser un desastre entonces me atrevo a coger ese trabajo y vamos y lo hago, o sea te da más seguridad (Isabel, familiar).

\subsection{FORMACIÓN BASADA EN EVIDENCIAS CIENTÍFICAS}

El análisis del trabajo de campo realizado aporta diversas evidencias que destacan las ventajas obtenidas cuando se han implementado actuaciones educativas de éxito tales como grupos interactivos (Valls \& Kyriakides, 2013) o tertulias literarias dialógicas (De Botton, Girbés, Ruiz \& Tellado, 2014) en formación de familiares. Así, y en relación a los grupos interactivos, profesorado diverso entrevistado ha indicado cómo esta forma de organización del aula ha posibilitado el obtener beneficios de la diversidad de niveles educativos y de perfiles de participantes que se encontraban en la misma aula y que inicialmente identificaban como un factor problemático. En la siguiente cita se recoge el cambio experimentado en el grupo de familiares tras la implementación de los grupos interactivos, respecto del año anterior, en el que no se había implementado aún esa forma organizativa del aula, explicado por el profesor del grupo:

El año pasado yo ya dije, es que nos metieron en la clase a todos los padres y súper mal, porque verás tú, hay padres que están con las restas y otros que no han empezado con las sumas y hay otros que ya están con las divisiones. Este año ya está solucionado, ya no es un problema $\left(\right.$ Pablo, profesor $\left.^{3}\right)$.

Todos los nombres que aparecen en las citas de este artículo son ficticios y protegen el anonimato de las personas que los han realizado. 
Por otro lado, y en relación al beneficio que las tertulias literarias dialógicas representan para el aprendizaje de los familiares adultos, destacamos la cita de una madre que participa en esta actuación educativa de éxito y donde destaca el impacto que este tipo de actuación ha generado tanto en el contexto escolar como en otros espacios de su vida familiar:

Sí que influye porque nosotros ya te digo, comentamos, pues igual que podemos hablar de una peli, ¿qué peli vemos esta semana? Hablamos de los clásicos universales, de los que leemos [...] Y no solo en mi casa, vamos a casa de la familia y todos me preguntan, ¿qué libro estáis leyendo? ¿cómo va la tertulia? [...] Pero sí, se habla de literatura, entonces sí que ha influido (Rosa, madre).

Otro elemento relevante tiene que ver con la inclusión de los principios del aprendizaje dialógico (Aubert et al., 2008) en la formación de familiares. El análisis de los datos confirma la influencia positiva de la aplicación de enfoques científicos como el del aprendizaje dialógico como base para entender el éxito de la formación de familiares. Las personas entrevistadas han hecho mención explícita a la importancia de aplicar los diversos principios de este paradigma, como por ejemplo el reconocimiento de la inteligencia cultural, el diálogo igualitario o la igualdad de diferencias.

En la siguiente cita correspondiente a una de las voluntarias en la formación de familiares se hace referencia a la importancia del reconocimiento de la inteligencia cultural para superar algunos de los obstáculos que dificultan los avances académicos de los y las familiares:

Ellos tienen un conocimiento propio adquirido de haber vivido esa pobreza, que no lo tenemos el resto, y eso muchas veces no se pone en alza, ese valor, ese conocimiento, no se valora a lo mejor en el resto de Sevilla, o por otras personas o incluso entre vecinos, yo creo que de ahí vienen muchos prejuicios, de no valorar eso (Ana María, voluntaria).

La participación genera una mayor participación y la superación de barreras personales y sociales. De este hecho también se han recogido numerosas evidencias, donde se ha identificado cómo en algunos centros han vinculado la formación de familiares con la promoción de la participación de colectivos vulnerables en iniciativas que se han realizado fuera de la escuela tales como la invitación a congresos sobre investigación educativa como ponentes. En la siguiente cita correspondiente a una madre que participa en la formación de familiares se recoge la importancia que tuvo para ella y para sus compañeras el poder asistir y participar por primera vez en sus vidas en un congreso, identificándolo como una muestra de superación personal:

Marga nos metió, pero de lleno. Nos llevaba a congresos para docentes, que decíamos, pero Marga ¿qué hacemos aquí, si somos madres? [...] ¿Cómo es posible? Y lo hacíamos. Y lo hacíamos como si lo hubiésemos hecho toda la vida. Y recuerdo el último congreso que se hizo en Valencia y habíamos tomado nuestros apuntes, habíamos dirigido unas mesas, y cuando llegó el momento de exponer los resúmenes y demás. Pues claro lo tenía que hacer cada profesora con su centro, y ella me dijo a mí, no lo voy a hacer yo, lo vas a exponer tú. Y yo dije, no, no, no me hagas esto, ¿cómo 
voy a hablar yo delante de 400 personas todos profesores? Digo, no me hagas eso. Y dice Marga, venga va que yo sé que tú te vas a atrever. Y yo dije, si he llegado hasta aquí ¿Por qué no? Me senté allí, todo el público mirando, y no sé si era yo la séptima o la octava, cuando me dieron el micrófono estaba asustadísima y dije, quiero dejar claro que solo soy una madre, que no soy docente, no tengo ni idea y no sé cómo lo voy a hacer. Claro, la gente me aplaudió para animarme un poco porque hasta allí no se había aplaudido a ningún profesor. Entonces ya dije, bueno, desde aquí camino ya hacía donde haga falta. Y esto sí que fue un trabajo de Marga, nos vimos capaces de lo que fuera (M. Teresa, madre).

La ruptura de barreras para incluir no solo las voces de las familias, sino que ellas mismas se hagan garantes y portavoces de los logros de su propia participación, ha contribuido según todas las familiares entrevistadas y la mayor parte de las voluntarias y maestras, a potenciar la participación en educación de personas adultas (y, también, a transformar la imagen "social" que sienten tener esas familias respecto de la educación).

\subsection{PROMOCIÓN DE OPORTUNIDADES PARA LA CONCILIACIÓN FAMILIAR}

Ampliar las oportunidades de conciliación familiar ha sido identificado como un factor relevante, sobre todo en relación a la participación de las mujeres en la formación de familiares. Este elemento cobra mayor relevancia si se tiene en cuenta los elevados índices de natalidad que suelen presentar las familias pertenecientes a colectivos vulnerables y sus escasas posibilidades para contar con otros servicios familiares extraescolares. Promover oportunidades para que estas mujeres puedan conciliar el cuidado de los hijos e hijas y la formación ha sido esencial para eliminar algunas de las barreras que tradicionalmente dificultan su acceso a la educación. Algunas de las estrategias identificadas en este sentido han sido la participación de las mujeres en las decisiones sobre los horarios y organización de la formación. La siguiente cita pertenece a una de las madres que participa en la formación de familiares en una escuela en la que las mujeres han decidido que el horario de la formación sea por las mañanas. Como se aprecia en la siguiente cita, el hecho de que la formación sea impartida en el mismo centro y en el mismo horario en el que sus hijos están en clase ha posibilitado que ella se involucre en las formaciones:

Es el colegio que van mis hijos y la hora que salimos y entramos es la misma que la de mis niños (Carmen, madre).

Otra de las estrategias identificadas para promover la conciliación es la apertura de espacios educativos gratuitos donde las mujeres pueden dejar a sus hijos e hijas mientas asisten a la formación. En la mayoría de los casos, este espacio se ha gestionado a través de redes de solidaridad como es el caso de la biblioteca tutorizada, sin que ello suponga un gasto adicional para las familias o para los centros. En la siguiente cita, correspondiente a una madre que participó en un grupo de discusión, se describen las ventajas que aporta la biblioteca tutorizada tanto para las familiares como para el refuerzo educativo de sus hijos e hijas.

Viene muy cómodo. Si vengo a estudiar yo y en casa tengo que dejar los nenes y mi marido no está, ¿Cómo lo hago? No puedo. Entonces, aquí por las tardes se podían 
quedar los nenes por la tarde haciendo los deberes, bien atendidos y nosotros podíamos estudiar. Esto es algo fundamental. Y, por ejemplo, había madres que tienen seis [hijos], ¿cómo te vas a poner a estudiar con seis en casa? Es que no puedes (Vicenta, madre).

Por otro lado, diversos centros que han participado en la investigación y no disponían de estos espacios familiares han activado otro tipo de estrategias para facilitar la participación de las madres. En la siguiente cita correspondiente a una de las profesoras participantes en un grupo de discusión se identifica como promueven que las familiares puedan participar en la formación y asistir acompañadas de sus hijos e hijas en el caso en que estas lo necesiten.

Vienen madres con el carro y los niños pequeños, que no sea este el impedimento, porque a veces tienen que estar por el niño pequeño y no. Pues vienen se sientan las madres, colocamos los carros, tenemos juguetes preparadas para los niños... que no sea un impedimento, al contrario (Susana, maestra).

Disponer, por tanto, de servicios de apoyo para "liberar" a las mujeres adultas para poder participar en actividades de formación, es quizás, uno de los aspectos más relevantes que contribuyen a transformar la participación de familiares.

\section{DISCUSIÓN Y CONCLUSIONES}

El objetivo de este artículo es discutir cuáles son los elementos que dificultan que las familias participen en programas de formación de familiares -algo que la literatura científica ha identificado como una de las formas de participación de las familias que más éxito tienen en la mejora de los resultados de los niños y de las niñas (Flecha, 2015; García \& Ríos, 2014)-; y qué elementos sirven precisamente para superar estas dificultades y lograr que las familias sí que participen en procesos formativos.

En líneas generales, los datos que se han obtenido en EDU-FAM sugieren que la participación de las familias en actividades de formación se explica por un compendio complejo de elementos, individuales y sociales, personales y comunitarios, que incluyen aspectos tales como el poder ayudar a los hijos con sus propios aprendizajes, el incremento de las oportunidades laborales, el poder participar en otras actuaciones educativas de éxito, participar en otros aspectos de la vida cívica de la comunidad o del contexto de que se acaba transformando referencia.

Después de analizar los datos recogidos a lo largo de tres años de trabajo en el proyecto EDU-FAM, hemos identificado cuatro elementos que las personas participantes en el estudio mencionan como aspectos clave en promover la participación de las familias en programas de formación.

En primer lugar, las familias se involucran en actividades de formación principalmente por tres motivos (ver Figura 1): por su propio deseo de aprender (aprender en general), para ayudar a sus hijos y para aprender ellos mismos/as las habilidades básicas. Estos tres elementos explican el $65 \%$ de la muestra, siendo el "ayudar a los hijos" la motivación principal, con un $26 \%$ de las respuestas obtenidas.

En ese sentido, lo que hemos identificado como "fomento del aprendizaje instrumental" (sección 3.1) es decir, ofertar una formación basada en estándares de calidad (currículum de 
máximos y de altas expectativas) explica el éxito que ha conseguido el proyecto EDU-FAM para involucrar a un alto número de familias en actividades de formación. Las familias se involucran en actividades de formación cuando saben que contendrán conocimientos de calidad. La expectativa de "ayudar a los hijos" ha sido identificada como una variable que explica la participación de las familias en actividades de formación (Hoover-Dempsey \& Sandler, 2005; Walker, Wilkins, Dallaire, Sandler \& Hoover-Dempsey, 2005). De acuerdo con la literatura previa, las variables que se suelen agrupar bajo epígrafes como motivational beliefs (Murray et al., 2014; Tuohilampi, 2016) sugieren que la autopercepción de las familias como incapaces de enseñar, las bajas expectativas adquiridas por autobiografías marcadas por la frustración y malos resultados académicos, la falta de self-efficacy limitan tanto la capacidad como la sensación y la motivación que tienen las familias para involucrarse en actividades de formación, principalmente con miras a ayudar a sus hijos (Kim, 2009; Koonce \& Harper, 2005). Los resultados obtenidos en EDU-FAM sugieren que una actuación que contribuye a transformar estas barreras (muchas veces autoimpuestas) es ofrecer actividades de formación basadas en estándares de calidad (y no como una opción de remedial education).

Por otro lado, la investigación previa ya ha identificado que otro de los motivos que dificultan que las familias participen en actividades de formación es la negativa de algunos maestros/as a invitarles debido a su propia frustración trabajando con estudiantes de clase socio-económica baja (Eccles \& Harold, 1993) o incluso por pensar que es precisamente la familia la fuente de los problemas académicos de los hijos (Griffith, 1998; Trotman, 2001). Los resultados de EDU-FAM sugieren que cuando se ofrece una oferta de formación basada en criterios de calidad, y con un enfoque de altas expectativas, familias vulnerables como las descritas por la investigación previa tienden también a participar y valorar muy positivamente la formación, tal y como se muestra en las citas de la sección 3.1 de este artículo.

Un segundo elemento que explica la participación de los adultos en actividades de formación es el obtener una acreditación que certifique su nivel educativo o su competencia en una materia en particular, sobre todo con miras a facilitarse su acceso al mercado laboral. Esta conclusión es consistente con los estudios previos en el ámbito (Brine, 2006; Jarvis, 2004; Morstain \& Smart, 1974; O’Donoghue \& Maguire, 2005). En este sentido, los datos recabados en EDU-FAM no hacen sino confirmar lo que ya se sabía: las personas adultas se apuntan a cursos de formación porque necesitan la titulación para mejorar su empleabilidad. Las citas de la sección 3.2 confirman este aspecto, que también es sobresaliente en los resultados del cuestionario: un 20\% de las personas que lo contestaron afirman que participan en actividades de formación para "aprender habilidades básicas" (la segunda categoría de respuesta, por orden de frecuencia relativa).

El tercer elemento identificado que explica la participación de las personas adultas en actividades de formación es la existencia de lo que hemos denominado "formación basada en evidencias científicas." Los datos recabados sugieren que es importante y necesario desarrollar la formación de familiares a partir de la puesta en práctica de actuaciones educativas que han evidenciado su eficacia como, por ejemplo, los grupos interactivos (García-Carrión y Díez-Palomar, 2015; Díez-Palomar y Cabré, 2015) o las tertulias literarias dialógicas (De Botton, Girbés, Ruiz \& Tellado, 2014; Pulido \& Zepa, 2010). La participación en ambas actuaciones educativas de éxito a través de figuras como el voluntariado suponen una oportunidad para muchas familias que deciden acercarse a los centros educativos para contribuir en el proceso de aprendizaje. Al hacerlo, algunas de 
las personas entrevistadas en EDU-FAM afirman que la participación, sea en los grupos interactivos, sea en las tertulias literarias dialógicas, se acaba convirtiendo en una puerta hacia su propia formación, porque en muchas ocasiones esas personas proponen y demandan formación para sí mismas. Esto expande la clasificación que tradicionalmente se había aceptado en este ámbito de estudio de las formas que tienen las familias para involucrase en la educación (Hoover-Dempsey \& Sandler, 2005; Peressini, 1998; Sanders \& Lewis, 2005). Por un lado, nuestros datos son consistentes con estudios previos que sugieren que no toda forma de participación de las familias conduce al éxito educativo de los estudiantes (Díez-Palomar y Kanes, 2012). A partir de los resultados obtenidos, hemos identificado aspectos transformadores de dichas actuaciones y aspectos exclusores de las mismas. Los grupos interactivos se han mostrado exitosos por los beneficios observados en el proceso de aprendizaje de los participantes, todos ellos con diversos niveles y perfiles educativos. Las tertulias literarias dialógicas se constituyen en actuaciones de éxito por la transformación de los referentes culturales y educativos que experimentan los participantes. Por tanto, la facilitación del aprendizaje y la capacidad de provocar cambios referenciales serían dos aspectos transformadores de la formación de familiares analizada.

Además, los resultados de EDU-FAM también sugieren otro elemento que también tiene potencial para explicar por qué las personas adultas participan en actividades de formación: la inclusión de sus voces. Las citas de la sección 3.3 muestran que cuando las personas adultas no solo participan en las actividades de formación, sino que además se les da oportunidad para explicar y hacer valer su experiencia, esto se traduce en un empoderamiento que redunda de manera directa en el sentido que tiene para ellos el participar en dichas actividades. En 1974, Morstain y Smart señalaron cinco factores que explican por qué las personas adultas participan en actividades de formación. Estos factores han sido confirmados por investigaciones posteriores. Sin embargo, ninguno de ellos incluye el reconocimiento de la voz de las personas adultas entre ellos; algo que tanto Freire (1998) como Flecha (2000) ya habían vaticinado desde sus respectivas teorías del aprendizaje que sucedería cuando se da la oportunidad a las personas adultas para participar inclusivamente. Los datos de EDU-FAM confirman con evidencias esta conclusión teórica previa.

Estudios previos en el ámbito del lifelong learning, de la participación de adultos en formación y de la formación de familiares sugieren que el perfil de "adulto" que participa en actividades de formación suele ser, por norma general, o bien la persona mayor de $55 \mathrm{o}$ 60 años de edad, mayoritariamente mujer, que ve sus responsabilidades reducidas cuando los hijos marchan de casa, o bien jóvenes que abandonaron el sistema educativo y regresan de nuevo en busca de certificaciones para mejorar sus expectativas laborales. En otros casos, cuando hablamos de "familias", Hoover y Dempsey (2005) mostraron que a pesar de que muchas familias se apuntarían a actividades de formación, no lo hacen por falta de tiempo, o por falta (real o auto-percibida) de conocimientos previos o sensación de que son capaces de aprender ellos mismos/as.

Sin embargo, los resultados de EDU-FAM aportan con un cuarto elemento necesario para superar estas situaciones descritas y facilitar que más familiares puedan participar en actividades de formación: la existencia de medidas de conciliación familiar. En la sección 3.4 se destaca que cuando los centros (o programas de formación) ofrecen soluciones para que las familias (madres fundamentalmente) puedan dejar atendidos a sus hijos durante el tiempo de la formación, entonces deciden participar activamente en dicha oferta formativa. 
En resumen, los cuatro elementos mencionados (con todos sus componentes) confirman, por un lado, variables que ya sabíamos que inciden en la participación de las familias en actividades de formación; pero, por otro lado, también sugieren nuevos aspectos a tener en cuenta, y que es necesario explorar más, seguramente por la vía cuantitativa, para encontrar evidencias que nos permitan generalizarlos y ampliar, de ese modo, las clasificaciones previas que existen sobre el tema. EDU-FAM ha contribuido a identificarlos. Ahora queda el trabajo de comprobar si son (o no) generalizables y transferibles a diversos contextos y países del mundo.

\section{REFERENCIAS BIBLIOGRÁFICAS}

Althusser, L. (1969). Para leer el Capital. México, D.F.: Siglo XXI. (v. o. 1965)

Aubert, A., Flecha, A., García, C., Flecha, R. \& Racionero, S. (2008). Aprendizaje dialógico en la Sociedad de la Información. Barcelona: Hipatia Press.

Aubert, A., Molina, S., Shubert, T. \& Vidu, A. (2017). Learning and inclusivity via Interactive Groups in early childhood education and care in the Hope school, Spain. Learning, Culture and Social Interaction, 13, 90-103. doi: 10.1016/j.lcsi.2017.03.002

Ausubel, D. P., Novak, J. D. \& Hanesian, H. (1989). Psicología Educativa. Un punto de vista cognoscitivo. México: Editorial Trillas. (v. o. 1978)

Baudelot, C. \& Establet, R. (1976). La educación capitalista en Francia. Madrid: Siglo XXI.

Boethel, M. (2004). Readiness. School, family \& community connections: Annual synthesis 2004. Austin, TX: Southwest Educational Development Laboratory.

Bourdieu, P. (1990). Artistic taste and cultural capital. In J.C. Alexander \& S. Seidman, Culture and society: Contemporary debates (pp. 205-215). Cambridge: Cambridge University Press.

Bourdieu, P. \& Passeron, J. C. (1977). La reproducción. Elementos para una teoría del sistema de enseñanza. Barcelona: Laia. (v. o. 1976)

Bowles, S. \& Gintis, H. (1985). La instrucción escolar en la América capitalista. Madrid: Siglo XXI. (v. o. 1976)

Brine, J. (2006). Lifelong learning and the knowledge economy: those that know and those that do not - the discourse of the European Union. British Educational Research Journal, 32(5), 649665. doi: 10.1080/01411920600895676

Castelli, S. \& Pepe, A. (2008). School-parents relationships: A bibliometric study on 40 years of scientific publications. International Journal about Parents in Education, 2(1), 1-12. Retrieved from http://www.ernape.net/ejournal/index.php/IJPE/article/viewFile/67/52

Civil, M. (2007). Building on community knowledge: An avenue to equity in Mathematics education. In N. Nasir \& P. Cobb (Eds.), Improving access to mathematics: Diversity and equity in the classroom, (pp. 105-117). New York, NY: Teachers College.

Civil, M. \& Andrade, R. (2003). Collaborative practice with parents: The role of researcher as mediator. In A. Peter-Koop, V. Santos-Wagner, C. Breen \& A. Begg (Eds.), Collaboration in teacher education: Examples from the context of mathematics education (pp. 153-168). Boston, MA: Kluwer.

Cole, M. (Ed.). (1988). Bowles and Gintis revisited: Correspondence and contradiction in educational theory. Lewes, Sussex, UK: The Falmer Press.

De Botton, L. Girbes, S., Ruiz, L. \& Tellado, I. (2014) Moroccan mothers' involvement in dialogic literary gatherings in a Catalan urban primary school: Increasing educative interactions and improving learning. Improving Schools 17(3), p. 241-249. doi: 10.1177/1365480214556420

De Graaf, N. D., De Graaf, P.M. \& Kraaykamp, G. (2000). Parental Cultural Capital and Educational Attainment in the Netherlands: A Refinement of the Cultural Capital Perspective. Sociology of 
Education, 73(2), 93- 111. Retrieved from http://www.jstor.org/stable/2673239

Desforges, C. (2003). The Impact of Parental Involvement, Parental Support and Family Education on Pupil Achievements and Adjustment. A Literature Review. London: Department for Educational Skills.

Díez-Palomar, J. \& Cabré, J. (2015). Using dialogic talk to teach mathematics: The case of interactive groups. ZDM, 47(7), 1299-1312. doi:10.1007/s11858-015-0728-x

Díez-Palomar, J. \& Kanes, C. (2012). Family and community in and out of the classroom: Ways to improve mathematics' achievement. Bellaterra, Barcelona: Servei de Publicacions de la Universitat Autònoma de Barcelona.

Díez-Palomar, J., Gatt, S. y Racionero, S. (2011). Placing immigrant and minority family and community members at the school's center: The role of community participation. European Journal of Education, 46(2), 184-196. doi:10.1111/j. 1465-3435.2011.01474.x

Driessen, G. \& Smit, F. (2007). Effect on immigrant parents' participation in society on their children's school performance. Acta Sociológica, 50(1), 39-56. doi: 10.1177/0001699307074882

Eccles J. S. \& Harold R. D. (1993). Parent-school involvement during the early adolescent years. Teachers College Record, 94, 568-587.

Epstein, J., Sanders, M., Simons, B., Salinas, K., Jansorn, N. \& Van Voorhls, F. (2002). School, Family and Community Partnerships. Your Handbook for Action. Thousand Oaks, CA: Corwin Press.

Flecha, R. (Ed.) (2015). Successful Educational Actions for Inclusion and Social Cohesion in Europe. Berlin: Springer. . (2000). Sharing words: Theory and practice of dialogic learning. Rowman \& Littlefield.

Flecha, R., Soler-Gallart, M. \& Sordé, T. (2015). Europe must fund social sciences, Nature, 528 (7581), 193. doi: 10.1038/528193d

Freire, P. (1998). Pedagogy of freedom: Ethics, democracy, and civic courage. Rowman \& Littlefield.

García-Carrión, R. \& Díez-Palomar, J. (2015). Learning communities: Pathways for educational success and social transformation through interactive groups in mathematics. European Educational Research Journal, 14(2), 151-166. doi: 10.1177/1474904115571793

García-Espinel, T. (2015). Contribuciones de la Mujer Gitana a la Ciencia, a las Políticas y a la Mejora Social. Multidisciplinary Journal of Gender Studies, 4(3), 832- 855. doi:10.4471/ generos.2015.1721

Garcia, L. B. \& Ríos, O. (2014). Participation and family education in school: Successful educational actions. Studiesin the Education of Adults,46(2),177-191.doi: 10.1080/02660830.2014.11661665

Gatt, S., Ojala, M. \& Soler, M. (2011). Promoting social inclusion counting with everyone: Learning Communities and INCLUD-ED. International Studies in Sociology of Education, 21(1), 33-47. doi:10.1080/09620214.2011.543851

Gómez, A., Puigvert, L. \& Flecha, R. (2011). Critical communicative methodology: Informing real social transformation through research. Qualitative Inquiry, 17(3), 235-245. doi: $10.1177 / 1077800410397802$

Gómez, A. \& Munté, A. (2015). Communicative methodology of research and Roma migrant women in Spain: A process of social change. In L. Bryant (Ed.), Critical and Creative Research Methodologies in Social Work (pp. 61-74). New York: Routledge.

Gómez, A., Munté, A. \& Sordé, T. (2014). Transforming Schools Through Minority Males' Participation: Overcoming Cultural Stereotypes and Preventing Violence. Journal of Interpersonal Violence, 29(11), 2002-2020. doi:10.1177/0886260513515949

Gómez, J. (2002). Learning Communities: When learning in common means school success for all. Multicultural Teaching, 20(2), 13-17.

González, N., Andrade, R., Civil, M. \& Moll, L.C. (2001). Bridging funds of distributed knowledge: Creating zones of practices in mathematics. Journal of Education for Students Placed at Risk, 6, 115-132. doi: 10.1207/S15327671ESPR0601-2_7 
Griffith J. (1998). The relation of school structure and social environment to parent involvement in elementary schools. The Elementary School Journal, 99, 53-80. doi: 10.1086/461916

Gutstein, E. (2007). Connecting community, critical, and classical knowledge in teaching mathematics for social justice. The Montana Mathematics Enthusiast, Monograph 1, 109-118. Retrieved from http://radicalmath.org/docs/Gutstein.pdf

(2006). Reading and writing the world with mathematics: Toward a pedagogy for social justice. New York: Routledge.

Hoover-Dempsey, K.V. \& Sandler, H.M. (2005). The Social Context for Parental Involvement: A path to Enhanced Achievement. Final Performance Report: OERI Grant \#R305T010673. Office of Educational Research and Innovation / Institute of Education Sciences, U.S. Department of Education.

INCLUD-ED Consortium (2009). Actions for Success in Schools in Europe. Brussels: European Commission.

Jarvis, P. (2004). Adult education and lifelong learning: Theory and practice. New York, NY.: Routledge.

Kim, Y. (2009). Minority parental involvement and school barriers: Moving the focus away from deficiencies of parents. Educational Research Review, 4, 80-102. doi: 10.1016/j. edurev.2009.02.003

Koonce D. A. \& Harper J. W. (2005). Engaging African American parents in the schools: A community-based consultation model. Journal of Educational and Psychological Consultation, 16, 55-74. doi: 10.1207/s1532768xjepc161\&2_4

Lastikka, A. L. \& García-Carrión, R. (2012). Participación de las familias en el currículo y la evaluación. Cuadernos de Pedagogía, 429, 61-63.

Morstain, B. R. \& Smart, J. C. (1974). Reasons for participation in adult education courses: A multivariate analysis of group differences. Adult education, 24(2), 83-98. doi: $10.1177 / 074171367402400201$

Murray, K. W., Finigan-Carr, N., Jones, V., Copeland-Linder, N., Haynie, D. L. \& Cheng, T. L. (2014). Barriers and facilitators to school-based parent involvement for parents of urban public middle school students. SAGE open, 4(4), doi: 10.1177/2158244014558030.

O’Donoghue, J. \& Maguire, T. (2005). The individual learner, employability and the workplace: a reappraisal of relationships and prophecies. Journal of European Industrial Training, 29(6), 436-446. doi: 10.1108/03090590510610236

Peressini, D. D. (1998). The portrayal of parents in the school mathematics reform literature: Locating the context for parental involvement. Journal for Research in Mathematics Education, 555-582.

Pulido, C. \& Zepa, B. (2010). La interpretación interactiva de los textos a través de las tertulias literarias dialógicas. Special Issue: Communicative acts for social inclusion, Signos, 43(2), 295309. Retrieved from http://www.scielo.cl/pdf/signos/v43s2/a03.pdf

Reale, E., Avramov, D., Canhial, K., Donovan, C., Flecha, R., Holm, P., Larkin, C., Lepori, B., Mosoni-Fried, J., Oliver, E., Primeri, E., Puigvert, L., Scharnhorst, A., Schubert, A., Soler, M., Soòs, S., Sordé, T., Travis, C. \& Van Horik, R. (2017). A review of literature on evaluating the scientific, social and political impact of social sciences and humanities research. Research Evaluation, 2017, 1-11. doi: 10.1093/reseval/rvx025

Reardon, S. (2011). The Widening Academic Achievement Gap Between the Rich and the poor: New evidence and possible explanations. In A. R. Murnane \& G. Duncan (Eds.), Whither Opportunity? Rising Inequality and the Uncertain Life Chances of Low-Income Children. New York: Russell Sage Foundation.

Sánchez Oñate, A., Reyes Reyes, F. \& Villarroel Henríquez, V. (2016) Participación y expectativas de los padres sobre la educación de sus hijos en una escuela pública. Estudios Pedagógicos, 42 (3), 347-367. doi: 10.4067/S0718-07052016000400019

Sanders, M. G. \& Lewis, K. C. (2005). Building Bridges Toward Excellence: Community Involvement 
in High Schools. The High School Journal, 88(3), 1-9. doi: 10.1353/hsj.2005.0005

SIOR, 2016. Social Impact Open Repository. Online access: http://www.ub.edu/sior/sior.php

SOJO (2011). Social Justice High School. Retrieved from http://sj.lvlhs.org

Soler-Gallart, M. (2017). Dialogic Relations and Interactions as an Alternative to Power. In Achieving Social Impact: Sociology in the Public Sphere (pp. 21-42). Cham: Springer International Publishing.

Suizzo, M. A. \& Stapleton, L. M. (2007). Home-based parental involvement in young children's education: examining the effects of maternal education across U.S. ethnic groups. Educational Psychology, 27, 533-556. doi: 10.1080/01443410601159936

Trotman, M. F. (2001). Involving the African American parent: Recommendations to increase the level of parent involvement within African American families. Journal of Negro Education, 70, 275-285. doi:10.2307/3211280

Tuohilampi, L. (2016). Contextualizing mathematics related affect: Significance of students' individual and social level affect in Finland and Chile. REDIMAT, 5(1), 7-27. doi: 10.4471/ redimat.2016.1823

Valls, R. \& Kyriakides, L. (2013). The power of Interactive Groups: how diversity of adults volunteering in classroom groups can promote inclusion and success for children of vulnerable minority ethnic populations. Cambridge Journal of Education, 43(1), 17-33. doi: 10.1080/0305764X.2012.749213

Vygotsky, L. S. (1996). El desarrollo de los procesos psicológicos superiores. Barcelona: Crítica (v. o. 1978)

. (1995). Pensamiento y lenguaje. Barcelona: Paidós. (v. o. 1934)

Walker J. M. T., Wilkins A. S., Dallaire J. R., Sandler H. M. \& Hoover-Dempsey K. V. (2005). Parental involvement: Model revision through scale development. The Elementary School Journal, 106, 85-104. doi: 10.1086/499193

Warren, M. R., Hong, S., Rubin, C. L. \& Uy, P. S. (2009). Beyond the bake sale: A community-based relational approach to parent engagement in schools. Teachers College Record, 111(9), 22092254 . 